\title{
Electrical transport in monolayers of phthalocyanine molecular wires and AFM imaging of a single wire bridging two electrodes
}

\author{
S. J. Tans, R. Miedema, L. J. Geerligs, and C. Dekker \\ Department of Applied Physics and DIMES, Delft University of Technology, Lorentzweg 1, 2628 CJ Delft, The Netherlands \\ J. Wu and G. Wegner \\ Max-Planck-Institüt fur Polymerforschung, P.O. Box 3148, W-55021 Mainz, Germany
}

\begin{abstract}
We report on several steps that are of interest for electrical transport studies on single molecular wires. Current-voltage measurements on monolayers of phthalocyaninepolysiloxane wires as a function of temperature are consistent with the spacecharge-limited-current model. Isolated wires are firmly immobilized on a substrate corrugated with metal electrodes by embedding them in a Langmuir-Blodgett monolayer of cellulose. High-resolution atomic-force-microscopy images have been obtained of a single molecular wire bridging two closely spaced electrodes.
\end{abstract}

Keywords: electrical transport measurements, Langmuir-Blodgett monolayers, single molecules, atomic force microscopy

\section{Molecular wires}

The issue of electron transport within single molecules is relevant for a number of scientific areas, ranging from the field of organic devices like FETs [1] and LEDs [2] to studies of electron transfer in proteins [3]. Furthermore, it is the central topic for possible electronic devices on a molecular scale [4]. Currently, various experimental routes are being explored to measure the conductivity of individual molecules [5]. Among the main experimental challenges are the realization of ultra-small electrodes to link the molecule to the macroscopic world, synthesis of suitable molecules, methods to deposit them in a controlled way, and imaging at the molecular scale. In this paper we will discuss results on these topics for our specific approach to measure electronic transport in a single molecular wire.

We study phthalocyaninepolysiloxane (PcPS) polymers [6] (see inset Fig.1). These are 20 to $50 \mathrm{~nm}$ long stacks of Si-phthalocyanine monomers linked by oxygen atoms. The monomer repeat distance of $0.33 \mathrm{~nm}$ generates orbital overlap between the monomers which accounts for the mechanical rigidity and renders the polymer a one-dimensional semiconductor.

\section{Electronic transport in monolayers of PcPS wires}

In order to make an evaluation of the conductivity of the PcPS wires, we have investigated the inplane electrical transport of monolayers of PcPS wires. Langmuir-Blodgett monolayers were deposited on interdigitated $\mathrm{Pt}$ electrodes with electrode spacings of $0.2 \mu \mathrm{m}$ and $1 \mu \mathrm{m}$. Figure 1 displays a set of current-voltage curves taken at different temperatures for a sample with $0.2 \mu \mathrm{m}$ spaced electrodes (dotted lines). The currents are two orders of magnitude higher than the leakage current from electrode to electrode and hysteresis was negligible. At low bias one can distinguish a linear $I-V$ relation. This is in line with Pt-PcPS contacts being ohmic, which can be expected from the work functions of the two materials ( $\sim 5.7 \mathrm{eV}$ and $\sim 5 \mathrm{eV}$ respectively). The data appear to be consistent with the space-charge-limitedcurrent (SCLC) model [7]. This model applies when the concentration of injected charge carriers exceeds the concentration of intrinsic charge carriers. The bias voltage $V_{x}$ marks the point where these two concentrations are equal. When $V \gg V_{x}$ space charge builds up which results in a power-law $I-V$ curve. The specific form of the $I-V$ relation depends on the trap distribution. A common approach is to consider an exponential trap distribution. A single analytical expression for both the ohmic and SCLC regime can be derived $[8,9]$. Fits of this formula (solid lines in Fig.1) appear to yield excellent agreement with the data except at the highest temperatures.

The conductivity appears to be low $\left(10^{-10} \mathrm{~S} / \mathrm{cm}\right.$ at RT). This follows from the considerable HOMO-LUMO gap of PcPS $(\sim 1.5 \mathrm{eV})$. In the undoped state, this results in a low concentration of charge carriers and corresponding low conductivity. The current through a single undoped molecule is therefore undetectably low $\left(10^{-18} \mathrm{~A}\right)$. Low conductivities 


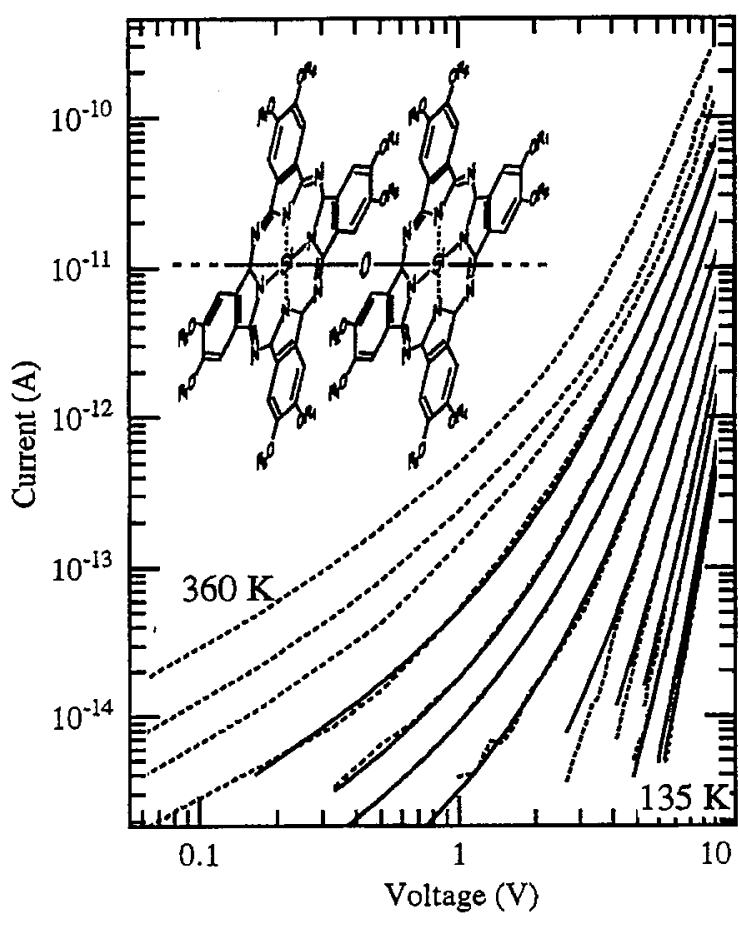

Figure 1: Inplane current through a monolayer of $P c P S$ wires as a function of applied voltage at various temperatures. Solid lines are fits to the space-charge-limited-current model. The inset is a schematic drawing of $\operatorname{PCPS}\left(\mathrm{R}_{1}=\mathrm{CH}_{3}\right.$, $\left.\mathrm{R}_{2}=\left(\mathrm{CH}_{2}\right)_{7} \mathrm{CH}_{3}\right)$.

are a general issue for experiments on single wires, since most considered molecules have a bandgap of at least $1 \mathrm{eV}$.

Chemical and electrochemical doping studies of the PcPS monolayers are currently underway. Preliminary results show that conductivity can be increased by at least 3 orders of magnitude. An alternative for increasing the conductivity may be the field effect. Field-effect gates have been realized by using highly doped Si wafers with a $100 \mathrm{~nm}$ layer of thermally grown oxide as substrates. The application of a gate voltage on a monolayer sample results only in a slight current increase up to about a factor of two. It is preceeded by large transient currents, that can not merely be accounted for by the different capacitors in the circuit. This may be related to remnant oxygen dopants that are mobile under the electric field.

\section{Electrical contacts to a single PcPS wire}

For any experiment on isolated single molecules as well as for the atomic force microscopy (AFM) imaging process, controlled deposition and immobilization of the molecules is crucial. Molecules that are adsorbed on a flat surface generally are mobile and will aggregate into clusters. In our work, the PCPS polymers are embedded in a Langmuir-Blodgett monolayer of insulating isopentylcellulose [10], resulting in a firm immobilization on the substrate. The LB layers can be deposited with various $\mathrm{PcPS} /$ cellulose ratios.

Figure 2 is an AFM image of four electrodes with a closest spacing of about $25 \mathrm{~nm}$, covered with a mixed

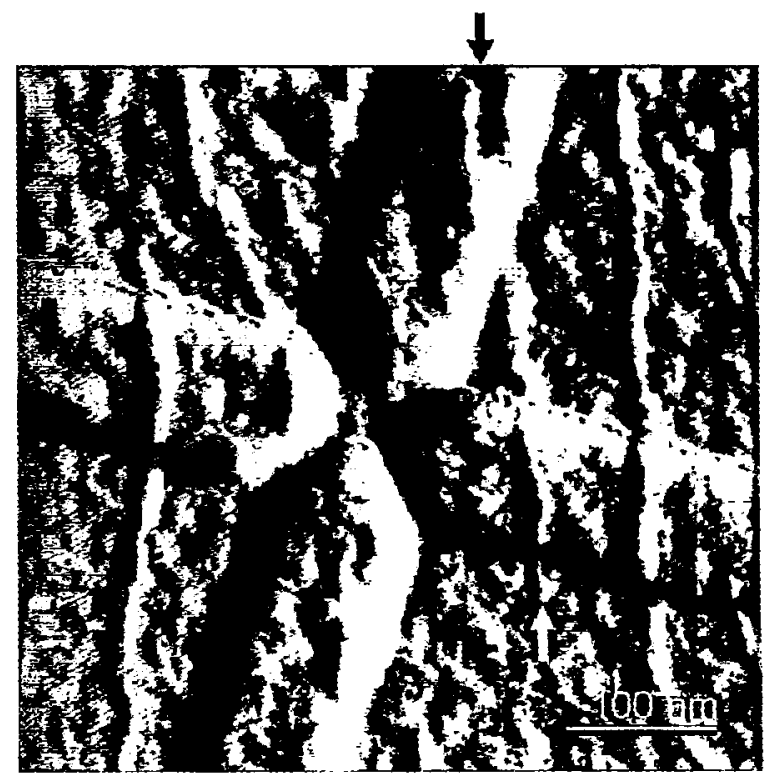

Figure 2: AFM image of a PCPS/cellulose monolayer on top of four Pt electrodes. The PcPS wire indicated by the arrows bridges two electrodes.

PcPS/cellulose monolayer. The PcPS molecules tend to order head-to-tail which accounts for apparent wire lengths of more than $100 \mathrm{~nm}$. The observed height of maximal $1.4 \mathrm{~nm}$ is consistent with the known diameter difference between PcPS and cellulose. The LB transfer apparently is not disturbed by the corrugation introduced by the electrodes. The PcPS wire indicated by the two arrows is seen to bridge two of the four electrodes.

\section{Acknowledgment}

We wish to acknowledge Dieter Neher and Hans Mooij for discussions. Research was supported by the Netherlands Foundation for Fundamental Research (FOM).

\section{References}

[1] F. Garnier, R. Hajlaoui, A. Yassar, P. Scivastava, Science 265 (1994) 1684.

[2] P. L. Burn, A. B. Holmes, A. Kraft, D. D. C. Bradley, A. R. Brown, R. H. Friend, and R. W. Gymer, Nature 356 (1992) 47.

[3] R. A. Marcus, N. Sutin, in Biochim. Biophys. Acta 811 (1985) 265.

[4] F. L. Carter, R. E. Siatkowski, and H. Wohltjen, Molecular Electronic Devices, (North Holland, 1988).

[5] Atomic and molecular wires, Ed. C. Joachim, NATOARW Conf. Proc. (Kluwer Acad. Publ, to be published).

[6] A. Ferencz, N. R. Armstrong, and G. Wegner, Macromolecules 27, 1517 (1994).

[7] M. A. Lampert and P. Mark, Current injection in Solids, Acad. Press, 1970.

[8] H. P. D. Lanyon, Phys. Rev. 130 (1963) 134.

[9] S. J. Tans, L. J. Geerligs, C. Dekker, J. Wu, and G. Wegner, to be published.

[10] J. Wu, G. Lieser, and G. Wegner, Adv. Mater. 8 (1996) 151. 